\title{
Pressure dependence of the boson peak in glasses
}

\author{
V. L. Gurevich \\ Solid State Physics Division, A. F. Ioffe Institute, 194021 Saint Petersburg, Russia \\ D. A. Parshin \\ Saint Petersburg State Polytechnical University, 195251 Saint Petersburg, Russia
}

H. R. Schober

Institut für Festkörperforschung, Forschungszentrum Jülich, D-52425 Jülich, Germany

(Received 21 July 2004; revised manuscript received 3 November 2004; published 28 January 2005)

\begin{abstract}
The inelastic scattering intensities of glasses and amorphous materials have a maximum at a low frequency, the so-called boson peak. Under applied hydrostatic pressure $P$, the boson peak frequency $\omega_{\mathrm{b}}$ is shifted upward. We have shown previously that the boson peak is created as a result of a vibrational instability due to the interaction of harmonic quasilocalized vibrations (QLV). By applying pressure one exerts forces on the QLV. These shift the low frequency part of the excess spectrum to higher frequencies. For low pressures we find a shift of the boson peak linear in $P$, whereas for high pressures the shift is $\propto P^{1 / 3}$. Our analytics is supported by simulation. The results are in agreement with the existing experiments.
\end{abstract}

DOI: 10.1103/PhysRevB.71.014209

PACS number(s): 61.43.Fs, 63.50+x, 78.30.Ly

\section{INTRODUCTION}

One of the most characteristic properties of glasses is a maximum in the low frequency part of their inelastic scattering intensities. ${ }^{1}$ This maximum, the boson peak (BP), originates from a maximum of the ratio $g(\omega) / \omega^{2}$ where $g(\omega)$ is the density of vibrational states which itself often has no corresponding maximum. ${ }^{2}$ The BP shows an excess of low frequency vibrations above the Debye contribution of the sound waves. It is observed in experiments on Raman scattering of light and inelastic neutron scattering within the frequency interval $0.5-2 \mathrm{THz}$ as well as in the temperature dependence of the specific heat. It is considered to be one of the universal properties of glasses and is found also in a number of other disordered systems-see Ref. 3 and references therein where also other approaches to the problem of the boson peak are discussed.

For the proper interpretation of the BP, the key problem is the nature of the vibrations that contribute to $g(\omega)$. Since the term BP frequently is used for any peak in the low frequency inelastic scattering intensity one has to distinguish between different cases. In some materials the BP is ascribed to low lying optical or transverse acoustic modes of parental crystals $^{4-6}$ or to librations of some molecules in plastic crystals. ${ }^{7,8}$ If these excitations have a small frequency spread they will show as a peak in $g(\omega)$. The role of disorder is merely to broaden modes which exist already without disorder.

In the opposite case the excess of low frequency modes is caused by disorder itself. A simple example is realized in a metalliclike model glass where the the parent crystal is fcc. ${ }^{9}$ In the present paper we discuss this latter case. Between these two extreme cases of well-defined low frequency modes, broadened by disorder, and no such modes before disorder, there is a range of materials having aspects of both. Despite being derived for the case of a disorder-induced BP, our results will apply, at least semiquantitatively, to these intermediate cases as long as the frequency spread of the relevant low frequency modes is sufficiently large.

Numerous explanations have been proposed for the origin of this disorder induced BP. It has been ascribed to vibrations of clusters of atoms of typical sizes ${ }^{10,11}$ which, however, so far have not been identified. Similarly, from random firstorder phase transition theory, rippling of domain boundaries was postulated as the origin of the BP. ${ }^{12}$ Another explanation, the broadening of acoustic sound waves as the origin of the BP (Refs. 13 and 14) is in contradiction with the linear dispersion law seen in molecular dynamics. ${ }^{15}$

A major effort has gone into the relationship between the $\mathrm{BP}$ and random matrices. Disorder affects the vibrational states differently than it affects the electronic ones. The main point is that the matrix determining the eigenvalues of the harmonic vibrations, the squared frequencies, must satisfy the requirement of mechanical stability (cf. Ref. 16). In other words, all eigenvalues must be positive, apart from the six zero values for translation and rotation of the system as a whole. Other than in the case of electronic states, there is a fixed zero level. An arbitrary random matrix has no such property. This means that, in general, such a matrix corresponds to an unstable vibrational system. Such a mechanical instability has often been observed in numerical simulations of disordered vibrational systems for sufficiently large degree of disorder (cf. Refs. 17-19).

However, as we have shown in Ref. 3, stability is restored automatically when the effects of anharmonicity are taken into account. A random matrix with the desired stability property is generated in a natural way by solving the corresponding nonlinear problem. It is remarkable that the density of states $g(\omega)$ of such a "stable matrix" possesses the BP feature. The BP is a reminder of the former mechanical instability in the system.

We start our investigation from the ubiquitously occurring quasilocal (or resonant) harmonic vibrations (QLV). These 
vibrations can be understood as a low frequency vibration of a small group of atoms which has a weak bilinear interaction with the continuum of acoustic vibrations of the whole system. They share many properties of the localized vibrations but are different from these exact harmonic eigenstates. They can be seen as resonances in the low frequency part of the local spectra of the set of atoms involved. In Mössbauer experiments one observes anomalously large Debye-Waller factors for the atoms vibrating with such QLV. ${ }^{20}$ In simulations the QLV are seen in the harmonic eigenstates as "low frequency localized modes" or mixed into the extended modes. This is found for the textbook case of a heavy mass defect as well as for QLV in glasses. ${ }^{21}$ A "low frequency localized mode" of a small system does not vanish when the system becomes larger-it just hybridizes with the other modes of similar frequency. If one counts the modes around the QLV frequency one still finds an additional eigenmode, compared to the modes in the unperturbed system. A more detailed discussion of QLV with emphasis on glasses can be found in our previous work on the boson peak ${ }^{3}$ where one can also find supporting references.

In the soft potential model ${ }^{22,23}$ one describes the low frequency vibrations of the defect system in the harmonic approximation utilizing a basis of (extended) sound waves and local oscillators. The latter are the cores of the QLV or "bare QLV" which have been found to extend over several atoms. ${ }^{24,25}$ In this basis there is a bilinear interaction which is treated as a perturbation.

\section{EFFECTS OF FINITE CONCENTRATION OF QUASILOCALIZED VIBRATIONS}

In a glass one has a finite concentration of QLV's. As discussed in our previous paper, ${ }^{3}$ this has a profound effect on their density of states (DOS). The interaction of the QLV's with the sound waves induces an elastic dipole interaction between them. First, the interaction of soft QLV's with surrounding QLV's of higher frequency may lead in harmonic approximation to unstable modes. Stability is restored by the anharmonic terms. This leads to a linear density of states, $g(\omega) \propto \omega$, for $\omega<\omega_{c}$ where $\omega_{c}$ is determined by the typical interaction strength.

Second, these renormalized low frequency QLV's interact with each other. In effect this means that the QLV's are subject to random forces $f$, later referred to as internal forces. Due to the high susceptibility of low frequency vibrations their low frequency DOS is changed to $g(\omega) \propto \omega^{4}$ for $\omega$ $<\omega_{\mathrm{b}}$. This is a general property of the low frequency DOS of non-Goldstone bosonic excitations in random media ${ }^{16}$ and has been discussed in numerous papers on low energy excitations in glasses, see Refs. 16, 23, 24, 26, and 27. It is associated with the so-called "seagull" singularity in the distribution of the stiffness constants of the QLV (Refs. 23 and 24) in the soft potential model. ${ }^{23}$ This seagull singularity has also been observed in computer simulations. ${ }^{28,29}$

By the crossover between the two limiting regimes of the DOS the BP is formed and obtains a "universal" shape. The frequency of the BP $\omega_{\mathrm{b}}$ is again determined by the interaction strength and thus by the characteristic value of the in- ternal forces $f_{0}$ (in Ref. 3 this quantity was denoted as $\delta f$ )

$$
\omega_{\mathrm{b}} \propto f_{0}^{1 / 3} .
$$

With higher interaction $\omega_{\mathrm{b}}$ is shifted upward and the intensity of the BP is reduced. ${ }^{3}$

One of the most interesting properties of the boson peak in glasses is its shift toward higher frequencies under application of hydrostatic pressure. In the present paper we will show that such a shift can be visualized as a result of a simple physical mechanism. If one applies a pressure $P$ onto a specimen of glass the internal random forces acquire additional random contributions $\Delta f$ proportional to the pressure. As a result, one obtains a "blue" shift of the Boson peak frequency with pressure. For small pressure this pressure contribution is small compared to the characteristic value of internal forces $f_{0}$ and the shift of the $\mathrm{BP}$ is linear in the pressure. With increasing pressure $\Delta f$ can become larger than $f_{0}$ and the BP shifts as $\omega_{\mathrm{b}} \propto P^{1 / 3}$.

\section{RANDOM FORCE DISTRIBUTION IN A GLASS UNDER PRESSURE}

In the present section we will briefly derive, in analogy to Ref. 3, the random force distribution under an applied hydrostatic pressure. From Hook's law one gets

$$
\varepsilon_{i k}=-(P / 3 K) \delta_{i k},
$$

where $\varepsilon_{i k}$ is the strain tensor and $1 / K$ is the compressibility of the glass.

The interaction of a QLV with the strain is bilinear ${ }^{30}$

$$
\mathcal{H}_{\text {int }}=\Lambda_{i k} \varepsilon_{i k} x=-(P / 3 K) \Lambda_{i i} x,
$$

where $\Lambda_{i k}$ is the deformation potential tensor and $x$ is the coordinate of the QLV. For simplicity we will write in the following $\Lambda$ instead of $\Lambda_{i i}$. Thus the additional contribution to the random force due to applied pressure is proportional to the pressure

$$
\Delta f=(P / 3 K) \Lambda .
$$

The deformation potential $\Lambda$ of a QLV is a random quantity. In particular, it has a random sign, so that the corresponding distribution function $D(\Lambda)$ is an even function of $\Lambda$. As a result, the distribution of the random forces $\tilde{f}$ in the glass remains an even function of $\widetilde{f}$ when the pressure is applied.

The total random force $\tilde{f}$ is a sum of two contributions

$$
\tilde{f}=f+\Delta f .
$$

Here $f$ is the internal random force in the absence of pressure. If the distribution of the internal forces $f$ is $Q(f)$ then the distribution of the total random force $\tilde{f}$ in a glass under pressure is given by the convolution

$$
F_{P}(\tilde{f})=\int_{-\infty}^{\infty} d \Lambda Q\left(\tilde{f}-\frac{\Lambda}{3 K} P\right) D(\Lambda) .
$$


For $P=0$ it reduces to the unperturbed distribution $Q(f)$ since the distribution $D(\Lambda)$ is normalized to unity. In the Appendix we give the results of the convolution for three cases which should be typical, namely, two types of Lorentzian distributions and a Gaussian distribution of $f$ and $\Lambda$.

\section{BOSON PEAK SHIFT UNDER PRESSURE}

Let us discuss how random forces change the frequencies of the QLV's. In a purely harmonic case, the linear forces would not affect the frequencies. Anharmonicity, however, renormalizes the relevant part of the spectrum. ${ }^{3,23}$ Although the QLV's are essentially harmonic vibrations their frequencies under applied forces can be shifted as in the usual quasiharmonic approximation.

To start the description of a QLV, consider an anharmonic oscillator under the action of a random static force $\tilde{f}$

$$
U(x)=A x^{4} / 4+M \omega_{1}^{2} x^{2} / 2-\tilde{f} x .
$$

Here $\omega_{1}$ is the oscillator frequency in the harmonic approximation and $A$ is the constant of anharmonicity. The role of the omitted third order term as well as that of the distribution of $A$ will be discussed further on. The force $\tilde{f}$ shifts the equilibrium position [the minimum of the potential $U(x)$ ] from $x=0$ to $x_{0} \neq 0$, given by

$$
A x_{0}^{3}+M \omega_{1}^{2} x_{0}-\tilde{f}=0,
$$

where the oscillator has a new harmonic frequency

$$
\omega_{\text {new }}^{2}=\omega_{1}^{2}+3 A x_{0}^{2} / M
$$

If $\widetilde{g_{1}}\left(\omega_{1}\right)$ is the distribution function of frequencies $\omega_{1}$ and $F_{P}(\tilde{f})$ is the distribution of random forces in a glass under pressure, then the pressure-dependent renormalized DOS is given by

$$
g_{P}(\omega)=\int_{0}^{\infty} \widetilde{g}_{1}\left(\omega_{1}\right) d \omega_{1} \int_{-\infty}^{\infty} d \widetilde{f} F_{P}(\widetilde{f}) \delta\left(\omega-\omega_{\text {new }}\right) .
$$

Integrating the $\delta$ function with regard to Eqs. (8) and (9) we get

$$
g_{P}(\omega)=2 M \sqrt{\frac{M}{3 A}} \omega^{3} \int_{0}^{\omega} \frac{\widetilde{g}_{1}\left(\omega_{1}\right) d \omega_{1}}{\sqrt{\omega^{2}-\omega_{1}^{2}}} F_{P}\left(f_{\omega, \omega_{1}}\right) .
$$

Here

$$
f_{\omega, \omega_{1}}=\frac{M}{3} \sqrt{\frac{M}{3 A}}\left(\omega^{2}+2 \omega_{1}^{2}\right) \sqrt{\omega^{2}-\omega_{1}^{2}} .
$$

As shown in Ref. 3 and shortly discussed in Sec. II the interaction of high and low frequency QLV lead to a linear DOS in the relevant frequency range. In writing Eq. (7) we took QLV already including this effect. Therefore, we can approximate $\widetilde{g_{1}}\left(\omega_{1}\right)=C \omega_{1}$ and write Eq. (11) in the form

$$
\begin{aligned}
g_{P}(\omega)= & 2 C M \sqrt{\frac{M}{3 A}} \omega^{4} \int_{0}^{1} \frac{d y y}{\sqrt{1-y^{2}}} \\
& \times F_{P}\left[\frac{M}{3} \sqrt{\frac{M}{3 A}} \omega^{3}\left(1+2 y^{2}\right) \sqrt{1-y^{2}}\right] .
\end{aligned}
$$

Let us introduce a characteristic value $\widetilde{f}_{0}(P)$ of the total random forces acting on the QLV's under pressure. This is the characteristic scale of variation of the argument of $F_{P}(\widetilde{f})$. Depending on the relative strengths of the internal forces without pressure and the pressure induced forces, the following estimates hold:

$$
\tilde{f}_{0}(P) \approx\left\{\begin{array}{c}
f_{0}\left[1+\mathcal{O}\left(P / P_{0}\right)\right] \text { for } P \ll P_{0} \\
\left(P / P_{0}\right) f_{0} \text { for } P \gg P_{0} .
\end{array}\right.
$$

Here $P_{0}$ is the characteristic pressure, which in the simple case of Lorentzian distributions centered around 0 [see Eqs. (A1) and (A2)], is given by $P_{0}=3 K f_{0} / \Lambda_{0}$. Thus for $P \ll P_{0}$, $\tilde{f}_{0}(P)=f_{0}$ while for $P \gg P_{0}, \tilde{f}_{0}(P) \propto P$.

According to Ref. 3, for a Lorentzian distribution of random forces, the Boson peak frequency is given by

$$
\omega_{\mathrm{b}}(P) \approx \frac{1.9 A^{1 / 6} \tilde{f}_{0}^{1 / 3}(P)}{M^{1 / 2}} .
$$

For frequencies $\omega \ll \omega_{\mathrm{b}}(P)$ the argument of the function $F_{P}$ in Eq. (13) is much smaller than the typical value of $\tilde{f}_{0}(P)$. One can replace this function by $F_{P}(0) \approx 1 / \widetilde{f}_{0}(P)$. According to Eq. (13), $g_{P}(\omega) \propto F_{P}(0) \omega^{4}$. Thus at high pressures $P \gg P_{0}$,

$$
g_{P}(\omega) \propto \omega^{4} / P \text { for } \omega \ll \omega_{\mathrm{b}}(P) .
$$

In the opposite case $\omega \gg \omega_{\mathrm{b}}(P)$ the integral over $y$ is dominated by such values of $y$ near the upper limit that

$$
\sqrt{1-y} \lesssim \frac{1}{M} \sqrt{\frac{A}{M}} \frac{\tilde{f}_{0}(P)}{\omega^{3}} \ll 1 .
$$

After integration, making use of the normalization of $F_{P}(\tilde{f})$, we regain the equation for the unperturbed pressureindependent linear density of states

$$
g_{P}(\omega)=\widetilde{g}_{1}(\omega) \propto \omega \text { for } \omega_{\mathrm{b}}(P) \ll \omega<\omega_{c}
$$

as it should be. For higher $\omega\left(\omega>\omega_{c}\right)$ the linear DOS produced by the interaction between the QLV will be modified and $g_{P}(\omega)$ shows material-dependent deviations.

Figure 1 shows the frequency dependence of the BP for different applied pressures. Both the distributions of internal forces $f$ and of the deformation potentials $\Lambda$ were approximated by Lorentzians centered at 0 . After convolution a broadened Lorentzian is obtained, Eq. (A3). One clearly observes the pronounced flattening with pressure of the low frequency part of the BP. Contrary to this, the high frequency part is not affected. In calculating the curves in Fig. 1 we assumed $\widetilde{g}_{1}(\omega) \propto \omega$. In real materials, this linearity of the DOS will only hold up to some frequency $\omega_{c}$. Above that frequency the DOS will be material dependent and, there- 


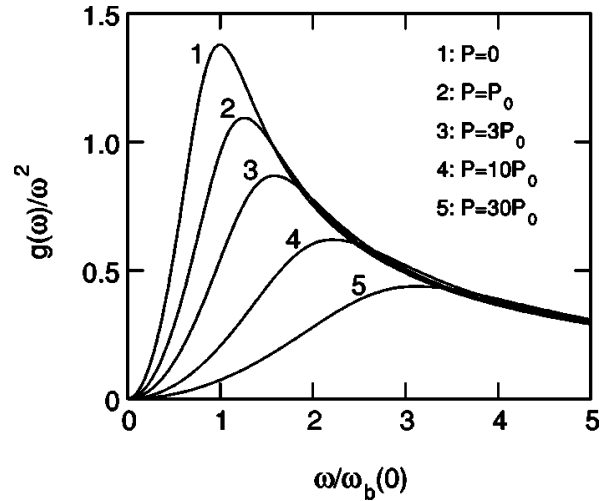

FIG. 1. Boson peak according to Eq. (13) for different applied pressures, excluding the low frequency Debye contribution. Lorentzian distributions were assumed for both internal and pressure induced forces, Eq. (A3).

fore, the BP will no longer have a universal form above that frequency. This can be seen by comparison of the present Fig. 1 with Fig. 3 of Ref. 3.

The variation of the BP frequency, $\omega_{\mathrm{b}}$, with pressure is shown in Fig. 2 for different distributions of the internal forces and deformation potentials. The limiting behavior for small and large pressures is independent of the distributions. The crossover, on the other hand, does somewhat depend on the type of distribution used. This indicates a material dependence in this pressure range. The shift of $\omega_{\mathrm{b}}$ with pressure can be described for the Lorentzian distributions, Eqs. (A1)-(A4), by

$$
\omega_{\mathrm{b}}(P)=\omega_{\mathrm{b}}(0)\left(1+\frac{|P|}{P_{0}}\right)^{1 / 3}
$$

with

$$
P_{0}=3 K f_{0} / \Lambda_{0} .
$$

and for the Gaussian distributions, Eqs. (A5)-(A8), by the slightly different form

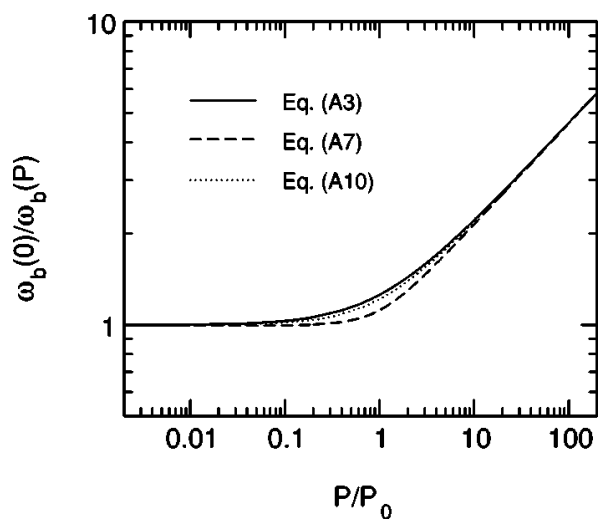

FIG. 2. Frequency $\omega_{\mathrm{b}}$ of the boson peak maximum as a function of applied pressure for different force distributions. Solid line: Lorentzian distributions Eq. (A3); dashed line: Gaussian distributions Eq. (A7); dotted line: double Lorentzian distribution, Eq. (A10), with $\lambda=\sqrt{2} \Lambda_{0}$.

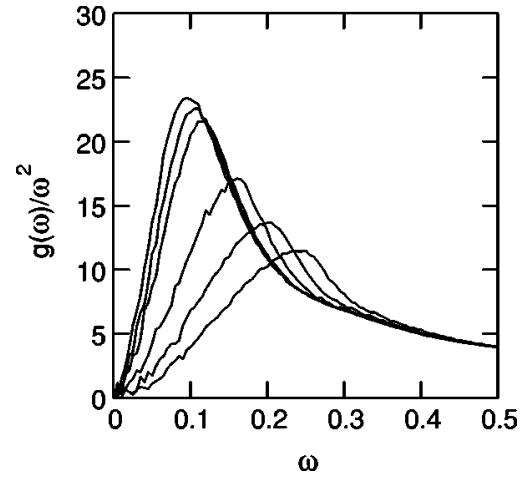

FIG. 3. Simulated $g(\omega) / \omega^{2}$ for different external force strengths $\left[N=2197, g_{0}(\omega) \propto \omega, J=0.07\right]$. Curves from left to right: $f_{\text {ext }}^{0}=10^{-4}$, $6 \times 10^{-4}, 10^{-3}, 3 \times 10^{-3}, 6 \times 10^{-3}$, and $10^{-2}$.

$$
\omega_{\mathrm{b}}(P)=\omega_{\mathrm{b}}(0)\left[1+\left(\frac{P}{P_{0}}\right)^{2}\right]^{1 / 6}
$$

which describes a sharper crossover.

\section{NUMERICAL SIMULATION}

\section{A. Pressure dependence}

To test our analytic description we extended the numerical simulations of Ref. 3 to include additional external forces $\Delta f$, Eq. (4). We placed $N=2197$ oscillators with frequencies $0<\omega_{i}<1$ on a simple cubic lattice with lattice constant $a$ $=1$ and periodic boundary conditions. The bilinear interaction between two oscillators, $i, j$, is written as

$$
U_{\text {int }}^{i j}=g_{i j}\left(J / r_{i j}^{3}\right) x_{i} x_{j},
$$

where $r_{i j}$ is the distance between the oscillators and $J$ is the strength of their coupling which results from the coupling of bare QLV's and sound waves, $J=\Lambda^{2} / \rho v^{2}$. Here $\rho$ is the density and $v$ the average sound velocity of the material. To simulate random orientations of the oscillators we took for $g_{i j}$ random numbers in the interval $[-0.5,0.5]$. The masses $M_{i}$ and anharmonicity parameters $A_{i}$ were put to 1 . The DOS for the noninteracting oscillators was taken as $g_{0}(\omega) \propto \omega^{n}$, with $n=1,2$. Random forces

$$
\Delta f^{i}=g_{i} f_{\mathrm{ext}}^{0}
$$

were exerted on the oscillators where $g_{i}$ were random numbers in the interval $[-0.5,0.5]$.

Generalizing the potential energy Eq. (7) for one oscillator to the system of $N$ oscillators and adding the interaction terms described by Eq. (22), we then minimized the potential energy of the total system of $N$ coupled anharmonic oscillators. In the usual harmonic expansion around this minimum we calculated the DOS for different values of $f_{\mathrm{ext}}^{0}$, representing different external pressures. This was repeated for up to 10000 representations.

The frequency dependence of $g(\omega) / \omega^{2}$ is given in Fig. 3, for $g_{0}(\omega) \propto \omega$ and $J=0.07$, for different strengths of the external force, $f_{\text {ext }}^{0}$. The behavior of the analytic results, Fig. 1, 


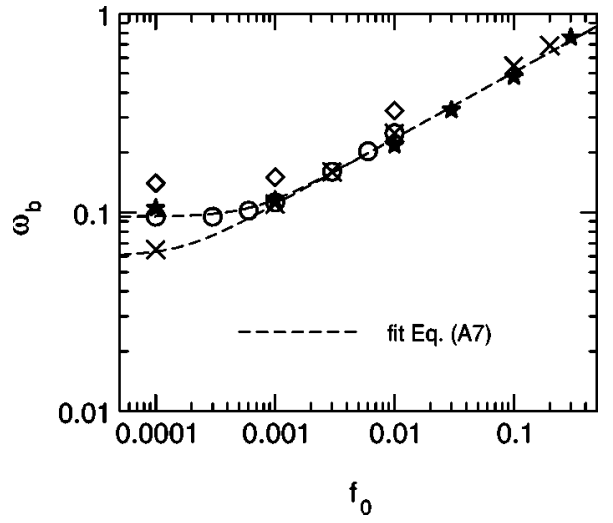

FIG. 4. Boson peak frequency $\omega_{\mathrm{b}}$ versus external force strength on a double logarithmic scale. Crosses: $N=2197, g_{0}(\omega) \propto \omega^{2}$, $J=0.1$; circles: $N=2197, g_{0}(\omega) \propto \omega, J=0.07$. The dashed lines give fits with Gaussian force distributions [fit parameters: $\omega_{\mathrm{b}}(0)$ and $P / P_{0}=1520 f_{\text {ext }}^{0}$ and $6010 f_{\text {ext }}^{0}$, respectively]. The asterisks and diamonds show the shift of the boson peak for weak and strong anharmonicity, as discussed further down.

is reproduced $\left(P / P_{0}=1520 f_{\text {ext }}^{0}\right)$. The slightly different maximal intensities for the higher forces (pressures) result from the different choice of distribution for the external forces (square instead of Lorentzian). The internal forces originate in the simulation directly from the bilinear interaction.

The pressure dependence of BP frequency is shown in a double logarithmic plot in Fig. 4 for two different original DOS's: $g_{0}(\omega) \propto \omega$ and $g_{0}(\omega) \propto \omega^{2}$. The results for both sets agree with our theoretical predictions. This illustrates that our results are indeed independent of the choice of the initial $g_{0}(\omega)$, as long as it is not too strongly peaked. As stressed in our previous work, above some frequency $\omega_{c}$ which is generally well separated above $\omega_{\mathrm{b}}$, the redistribution of frequencies becomes ineffective and the original $g_{0}(\omega)$ survives. For instance in a plot of $g(\omega) / \omega^{2}$, corresponding to Fig. 3, for $g_{0}(\omega) \propto \omega^{2}$ the curves converge to a constant given by the normalization, and the maximal intensities decay more slowly.

\section{B. Oscillator participation numbers}

In our model the formation of the BP is driven by the interaction between soft oscillators (bare QLV's). At low frequencies this interaction is weak and, therefore, the QLV's will be only weakly coupled. The BP frequency is determined by the typical interaction strength between the oscillators. To quantify the interaction we introduce an oscillator participation number

$$
n_{\mathrm{osc}}(\omega)=\left\langle\left(\sum_{j}\left|e^{j}\right|^{4}\right)^{-1}\right\rangle,
$$

where $e^{j}$ denotes the component on oscillator $j$ of an eigenvector of the coupled oscillator system and \langle\rangle$_{\omega}$ indicates the average over all eigenmodes of frequency $\omega$. Note that this oscillator participation number is different from the usual (atomic or molecular) participation number of an eigenmode

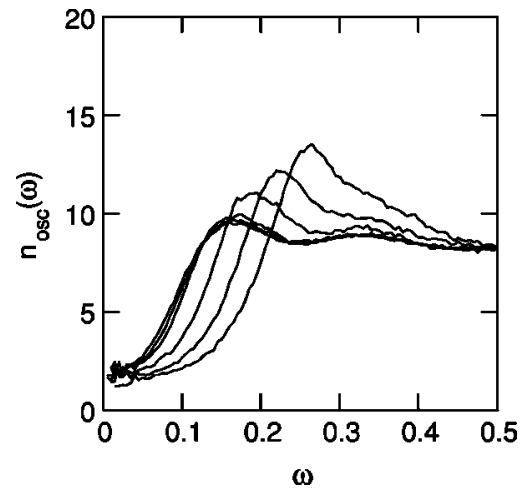

FIG. 5. Average oscillator participation numbers as function of frequency for different external force strengths. The curves correspond to the systems of Fig. 3.

of an atomic system. First a QLV (an oscillator in the present description) has typically an atomic participation number of ten or more. ${ }^{24,25}$ An oscillator participation number of ten is then equivalent to an atomic participation number of a hundred or more. Second the participation numbers are further increased by the interaction between the QLV's and the sound waves. ${ }^{21}$ Here this hybridization is only included insofar as it brings about the interaction between QLV's.

For all values of the applied external force $f_{\mathrm{ext}}^{0}, n_{\mathrm{osc}}(\omega)$ shows in Fig. 5 the same qualitative behavior as function of $\omega$. For small frequencies, one has more or less isolated QLV's $\left[n_{\text {osc }}(\omega) \approx 2\right]$. With increasing frequencies, coupling and hence $n_{\text {osc }}(\omega)$ rapidly increases. It reaches its maximum around $\omega_{\mathrm{b}}$ and drops to a plateau with $n_{\text {osc }}(\omega) \approx 8$. This might at first sight look surprising, since the coupling between the oscillators, Eq. (22) was not changed. On closer inspection of the coupled equations of motion and the equilibrium condition, one sees, however, that the external force does in fact change the coupling between the single oscillators. The maximal value of $n_{\text {osc }}(\omega)$ increases with $f_{\text {ext }}^{0}$ opposite to $g\left(\omega_{\mathrm{b}}\right) / \omega_{\mathrm{b}}^{2}$. This is what one would intuitively expect from an increased coupling. As in the case of the DOS, also $n_{\text {osc }}(\omega)$ depends on the original DOS, $g_{o}(\omega)$, for frequencies $\omega>\omega_{c}>\omega_{\mathrm{b}}$.

\section{Distribution of anharmonicity parameters}

So far, we have taken the anharmonicity parameter $A$ in Eq. (7) as a constant and have neglected possible third order terms. To check the influence of distributions of these terms, we did additional simulations where we introduced distributions of these parameters. The scaled results are summarized in Fig. 6, where we also show for comparison the theoretical result of Eq. (11) (dotted line). The solid line gives the simulation results for $g_{0}(\omega) \propto \omega$ and $J=0.07$ with a fixed value $A=1$. The simulated BP is slightly wider than the theoretical prediction which might be due to the non-Lorentzian distribution of the couplings between the "naked" oscillators [Eq. (7)]. The deviation at $\omega / \omega_{\mathrm{b}} \approx 2$ indicates the upper limit $\omega_{c}$ where the interaction strength no longer suffices to destroy 


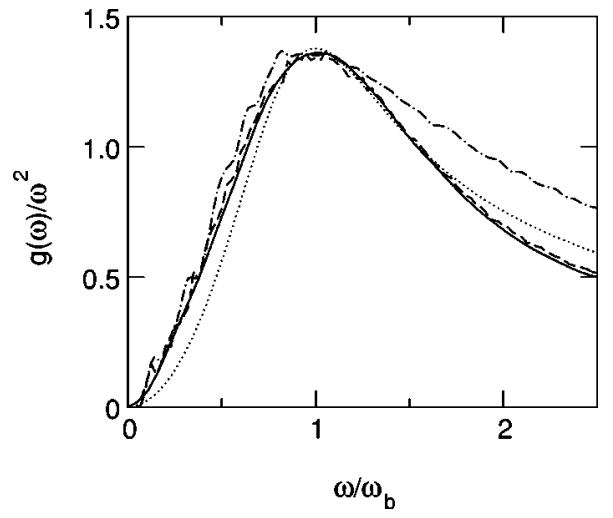

FIG. 6. Simulated, scaled boson peak for different anharmonicities $\left[N=2197, g_{0}(\omega) \propto \omega, J=0.07\right]$. Solid line: $B_{i}=0$; dashed line: weak anharmonicity; dash-dotted line: strong anharmonicity; dotted line: theoretical result.

the assumed density of the non-interacting oscillators, $g_{0}(\omega) \propto \omega^{2}$.

Taking, for the same parameters, random values $A_{i}$ from the interval $[0.7,1.3]$, no significant change can be discerned. This is in agreement with our previous result ${ }^{3}$ that this anharmonic term provides the mechanism to stabilize the interacting oscillators but, its magnitude does not determine the resulting spectrum.

The situation is different when we add a third order term to the energy of the single oscillators Eq. (7),

$$
U_{i}(x)=A x^{4} / 4+B_{i} x^{3} / 3+M \omega_{i}^{2} x^{2} / 2 .
$$

We obtain different behavior for weak and strong anharmonicy. The dashed line in Fig. 6 shows the resulting BP for weak anharmonicity with $B_{i}=b_{i} \omega_{i}$ with $b_{i}$ a random number from $[-1,+1]\left(g_{0}(\omega) \propto \omega\right)$. In this case Eq. (25) still describes single well oscillators. The shape of the BP is only marginally altered and the peak position for weak external forces (pressures) is slightly shifted (asterisks in Fig. 4). This weak effect can be understood considering that the term $B_{i} x^{3} / 3$ can be eliminated by a shift of origin resulting in a linear term representing an additional force on the oscillator.

For strong anharmonicity $\left(B_{i}^{2}>4 A M \omega_{i}^{2}\right) x=0$ in Eq. (25) no longer is a minimum but becomes the maximum of a double well potential. Under the influence of internal and external forces the oscillator can jump from one minimum to the other. Such a jump changes both the "naked" frequency of the oscillator and the dipole forces exacted on the other oscillators. This can no longer be described by a simple addition of forces. The resulting broadening of the BP is considerably increased and it will be shifted. The dash-dotted line in Fig. 6 shows this for $B_{i}=0.5 b_{i}$ with $b_{i}$ a random number from $[-1,+1]$. For this choice of parameters one has a large number of double well potentials, much higher than can be expected in real glasses. Figure 4 (diamonds) shows a sizable frequency shift for this case. Nevertheless, under an applied external pressure the shift of the BP with pressure follows again the analytic prediction.

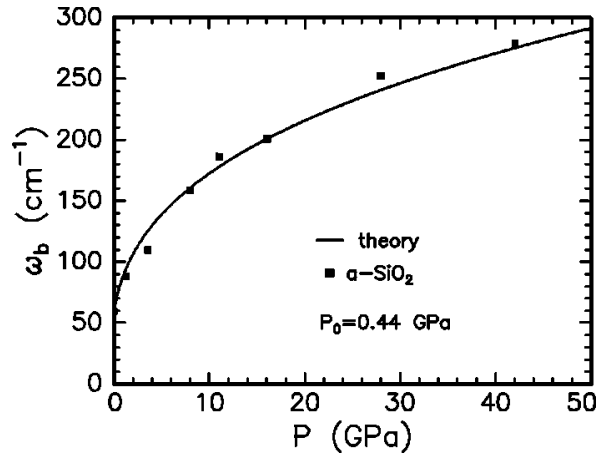

FIG. 7. The boson peak in $a-\mathrm{SiO}_{2}$ under pressure; filled squares are data of Ref. 39.

\section{COMPARISON WITH EXPERIMENT}

Unfortunately not too many experimental data are available. Our theory should, therefore, be considered rather as a prediction concerning future experiments than as an interpretation of the existing experimental data. A general increase of $\omega_{\mathrm{b}}$ has been observed in experiments on a number of materials, e.g., $\mathrm{SiO}_{2},{ }^{31,32} \mathrm{GeO}_{2},{ }^{31} \mathrm{GeS}_{2},{ }^{33}$ polybutadiene, ${ }^{34}$ polystyrene, ${ }^{35}$ and Teflon. ${ }^{36}$ Similar shifts have been reported from computer simulations of $\mathrm{SiO}_{2} \cdot{ }^{37,38}$ However most of these data are not sufficient for a quantitative analysis.

The shift of the BP over a large pressure range has been measured in $a-\mathrm{SiO}_{2} \cdot{ }^{39}$ As shown in Fig. 7, the experimental data can be fitted by our theory using Eqs. (19) and (20) and assuming a Lorentzian distribution [see Eq. (A3)]. The agreement between the theory and experiment remains good even for high pressures. Regarding very high pressures, our theory is applicable as long as the short range topology that determines the structure of QLV's does not change.

Figure 8 shows the shift of the boson peak in $a-\mathrm{GeS}_{2}$ measured by Raman scattering. Although the scatter of the experimental points is rather large, again the general agreement with our theory is encouraging.

Recent measurements on polyisobutadiene ${ }^{40}$ show an increase of the BP frequency $\propto p^{1 / 3}$. Boyer et al. ${ }^{36}$ measured the shift with pressure of the low temperature maximum $C(T) / T^{3}$ in Teflon, where $C(T)$ is the specific heat. This maximum is directly related to the $\mathrm{BP} .^{41}$ Again the observed shift fits well with our predictions, see Fig. 9.

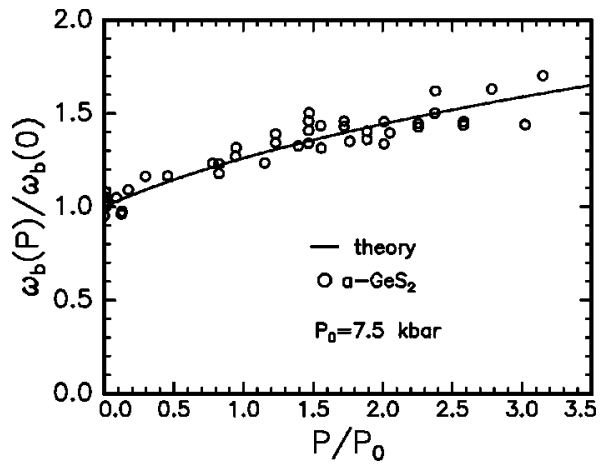

FIG. 8. The boson peak in $a-\mathrm{GeS}_{2}$ under pressure; open circles are data of Ref. 33. 


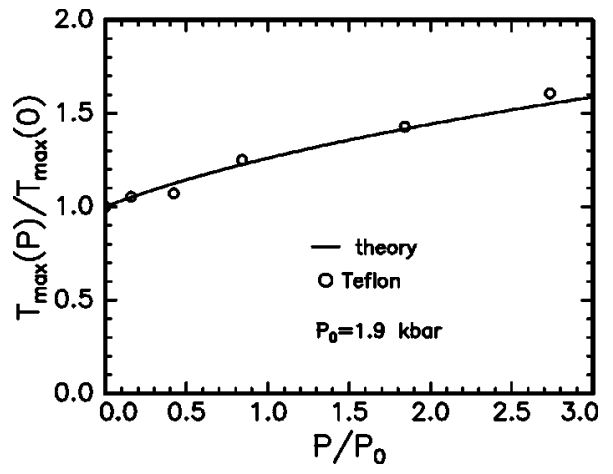

FIG. 9. The position of the bump $T_{\max }(P)$ in the specific heat $C(T) / T^{3}$ in Teflon under pressure; open circles are the data of Ref. 36.

However, the experiments on the change of the boson peak position under pressure are so far insufficient. Therefore, we believe that further detailed investigations of this phenomenon are called for.

\section{CONCLUSION}

In our previous paper $^{3}$ we have proposed a mechanism of the boson peak formation. The essence of the mechanism is that a vibrational instability of the spectrum of weakly interacting QLV is responsible for the origin of the boson peak in glasses and some other disordered systems. Anharmonicity stabilizes the structure but does not determine the shape of the boson peak. The vibrations forming the boson peak are harmonic.

The present paper extends these ideas. We show that under the action of hydrostatic pressure the boson peak is shifted to higher frequencies. At comparatively low pressures the shift is linear in pressure $P$ while for high pressures it is proportional to $P^{1 / 3}$. These conclusions are in good agreement with the existing experimental data. Our work explains the shift of the boson peek without the need to postulate additional negative third order anharmonicities. ${ }^{42}$

To obtain a quantitative proof, more extensive investigations of the pressure dependence of the boson peak position in various disordered systems are needed. Since the proposed mechanism is very general, it will also be interesting to investigate both theoretically and experimentally the behavior of the boson peak under different types of strain other than the hydrostatic one, studied here, as well as under static electric fields. In future work, we hope to show that the same physical mechanism is fundamental not only for the formation of the Boson peak but also for such seemingly different phenomena as the creation of the two level systems that dominate the properties of glasses at low temperatures.

\section{ACKNOWLEDGMENTS}

Two of the authors, V.L.G. and D.A.P., gratefully acknowledge the financial support of the German Ministry of Science and Technology and the hospitality of the Forschungszentrum Jülich where part of the work was done.
V.L.G. also acknowledges the financial support of the Alexander von Humboldt Foundation and of the Russian Foundation for Basic Research under Grant No. 03-02-17638.

\section{APPENDIX: CONVOLUTION}

To make our investigation more general, we will consider Lorentzian and Gaussian distributions for both the random forces and the deformation potential. Besides, we will consider also a distribution of the deformation potential $\Lambda$ that may be called "double Lorentzian." This is formed by two superimposed Lorentzian distributions with widths $\Lambda_{0}$ which are centered at $\pm \lambda$, respectively. For $\lambda>\Lambda_{0} / \sqrt{3}$ the resulting distribution then has two symmetric side maxima and a minimum at $\Lambda=0$.

For the Lorentzian distributions centered at zero one has

$$
Q(f)=\frac{1}{\pi} \frac{f_{0}}{f^{2}+f_{0}^{2}}=\frac{1}{2 \pi} \int_{-\infty}^{\infty} d \tau_{1} e^{i f \tau_{1}-f_{0}\left|\tau_{1}\right|}
$$

and

$$
D(\Lambda)=\frac{1}{\pi} \frac{\Lambda_{0}}{\Lambda^{2}+\Lambda_{0}^{2}}=\frac{1}{2 \pi} \int_{-\infty}^{\infty} d \tau_{2} e^{i \Lambda \tau_{2}-\Lambda_{0}\left|\tau_{2}\right|} .
$$

As a result of the convolution of these distributions one gets again a Lorentzian centered at zero but with greater width

$$
F_{P}(f)=\frac{1}{\pi} \frac{\widetilde{f}_{0}(P)}{f^{2}+\widetilde{f}_{0}^{2}(P)},
$$

where

$$
\tilde{f}_{0}(P)=f_{0}+\frac{\Lambda_{0}|P|}{3 K} .
$$

In the same manner the convolution of two Gaussian distributions

$$
Q(f)=\frac{1}{f_{0} \sqrt{2 \pi}} e^{-f^{2} / 2 f_{0}^{2}}=\frac{1}{2 \pi} \int_{-\infty}^{\infty} d \tau_{1} e^{i f \tau_{1}-f_{0}^{2} \tau_{1}^{2} / 2}
$$

and

$$
D(\Lambda)=\frac{1}{\Lambda_{0} \sqrt{2 \pi}} e^{-\Lambda^{2} / 2 \Lambda_{0}^{2}}=\frac{1}{2 \pi} \int_{-\infty}^{\infty} d \tau_{2} e^{i \Lambda \tau_{2}-\Lambda_{0}^{2} \tau_{2}^{2} / 2}
$$

leads to another Gaussian distribution with increased width.

$$
F_{P}(f)=\frac{1}{\tilde{f}_{0}(P) \sqrt{2 \pi}} \exp \left[-f^{2} / 2 \widetilde{f}_{0}^{2}(P)\right]
$$

where

$$
\tilde{f}_{0}(P)=\sqrt{f_{0}^{2}+\left(\frac{\Lambda_{0} P}{3 K}\right)^{2}}
$$


Finally let us convolute the Lorentzian for the internal forces, Eq. (A1), with a double Lorentzian distribution

$$
D(\Lambda)=\frac{1}{2 \pi}\left[\frac{\Lambda_{0}}{(\Lambda-\lambda)^{2}+\Lambda_{0}^{2}}+\frac{\Lambda_{0}}{(\Lambda+\lambda)^{2}+\Lambda_{0}^{2}}\right] .
$$

As a result of the convolution one gets

$$
F_{P}(f)=\frac{\tilde{f}_{0}(P)}{2 \pi}\left[\frac{1}{(f+\alpha \lambda)^{2}+\tilde{f}_{0}^{2}(P)}+\frac{1}{(f-\alpha \lambda)^{2}+\tilde{f}_{0}^{2}(P)}\right],
$$

where $\alpha=P / 3 K$ and $\tilde{f}_{0}(P)$ is given by Eq. (A4). This distribution now depends both on the widths of the two Lorentzians and on the distance between their centers, $2 \lambda$, in $D(\Lambda)$.
${ }^{1}$ Amorphous Solids. Low Temperature Properties, edited by W. A. Phillips (Springer-Verlag, Berlin, 1981).

${ }^{2}$ N. Ahmad, K. W. Hutt, and W. A. Phillips, J. Phys. C 19, 3765 (1986).

${ }^{3}$ V. L. Gurevich, D. A. Parshin, and H. R. Schober, Phys. Rev. B 67, 094203 (2003).

${ }^{4}$ Y. Tezuka, S. Shin, and M. Ishigame, Phys. Rev. Lett. 66, 2356 (1991).

${ }^{5}$ M. T. Dove, M. J. Harris, A. C. Hannon, J. M. Parker, I. P. Swainson, and M. Gambhir, Phys. Rev. Lett. 78, 1070 (1997).

${ }^{6}$ V. N. Sigaev, E. N. Smelyanskaya, V. G. Plotnichenko, V. V. Koltashev, A. A. Volkov, and P. Pernice, J. Non-Cryst. Solids 248, 141 (1999).

${ }^{7}$ M. A. Ramos, S. Vieira, F. J. Bermejo, J. Dawidowski, H. E. Fischer, H. Schober, M. A. González, C. K. Loong, and D. L. Price, Phys. Rev. Lett. 78, 82 (1997).

${ }^{8}$ R. M. Lynden-Bell and K. H. Michel, Rev. Mod. Phys. 66, 721 (1994)

${ }^{9}$ H. R. Schober, J. Phys.: Condens. Matter 16, S2659 (2004).

${ }^{10}$ V. K. Malinovsky, V. N. Novikov, and A. P. Sokolov, Phys. Lett. A 153, 63 (1991).

${ }^{11}$ T. Pang, Phys. Rev. B 45, 2490 (1992).

${ }^{12}$ V. Lubchenko and P. G. Wolynes, Proc. Natl. Acad. Sci. U.S.A. 100, 1515 (2003).

${ }^{13}$ S. R. Elliott, Europhys. Lett. 19, 201 (1992).

${ }^{14}$ C. Masciovecchio, V. Mazzacurati, G. Monaco, G. Ruocco, T. Scopigno, F. Sette, P. Benassi, A. Consulo, A. Fontana, M. Kirsch, A. Mermet, M. Montagna, F. Rossi, M. Sampoli, G. Signorelli, and R. Verbeni, Philos. Mag. B 79, 2013 (1999).

${ }^{15}$ S. N. Taraskin and S. R. Elliott, Phys. Rev. B 61, 12017 (2000).

${ }^{16}$ V. Gurarie and J. T. Chalker, Phys. Rev. Lett. 89, 136801 (2002); Phys. Rev. B 68, 134207 (2003).

${ }^{17}$ W. Schirmacher, G. Diezemann, and C. Ganter, Phys. Rev. Lett. 81, 136 (1998).

${ }^{18}$ T. S. Grigera, V. Martin-Mayor, G. Parisi, and P. Verrocchio, J. Phys.: Condens. Matter 14, 2167 (2002).

${ }^{19}$ S. N. Taraskin, J. J. Ludlam, G. Natarajan, and S. R. Elliott, Philos. Mag. B 82, 197 (2002).

${ }^{20}$ W. Petry, G. Vogl, and W. Mansel, Z. Phys. B: Condens. Matter
46, 319 (1982).

${ }^{21}$ H. R. Schober and G. Ruocco, Philos. Mag. 84, 1361 (2004).

${ }^{22}$ V. G. Karpov, M. I. Klinger, and F. N. Ignat'ev, Zh. Eksp. Teor. Fiz. 84, 760 (1983) [Sov. Phys. JETP 57, 439 (1983)].

${ }^{23}$ M. A. Il'in, V. G. Karpov, and D. A. Parshin, Sov. Phys. JETP 65, 165 (1987).

${ }^{24}$ U. Buchenau, Yu. M. Galperin, V. L. Gurevich, and H. R. Schober, Phys. Rev. B 43, 5039 (1991).

${ }^{25}$ B. B. Laird and H. R. Schober, Phys. Rev. Lett. 66, 636 (1991); H. R. Schober and B. B. Laird, Phys. Rev. B 44, 6746 (1991).

${ }^{26}$ I. L. Aleiner and I. M. Ruzin, Phys. Rev. Lett. 72, 1056 (1994).

${ }^{27}$ M. M. Fogler, Phys. Rev. Lett. 88186402 (2002).

${ }^{28}$ A. Heuer and R. J. Silbey, Phys. Rev. B 53, 609 (1996).

${ }^{29}$ H. R. Schober and C. Oligschleger, Phys. Rev. B 53, 11469 (1996).

${ }^{30}$ U. Buchenau, Yu. M. Galperin, V. L. Gurevich, D. A. Parshin, M. A. Ramos, and H. R. Schober, Phys. Rev. B 46, 2798 (1992).

${ }^{31}$ S. Sugai and A. Onodera, Phys. Rev. Lett. 77, 4210 (1996).

${ }^{32}$ Y. Inamura, M. Arai, M. Nakamura, T. Otomo, N. Kitamura, S. M. Bennington, A. C. Hannon, and U. Buchenau, J. Non-Cryst. Solids 293, 389 (2001).

${ }^{33}$ M. Yamaguchi, T. Nakayama, and T. Yagi, Physica B 263-265, 258 (1999).

${ }^{34}$ B. Frick and C. Alba-Simionesco, Appl. Phys. A: Mater. Sci. Process. 74, S549 (2002).

${ }^{35}$ R. Geilenkeuser, Th. Porschberg, M. Jäckel, and A. Gladun, Physica B 263-264, 276 (1999).

${ }^{36}$ J. D. Boyer, J. C. Lasjaunias, R. A. Fisher, and N. E. Phillips, J. Non-Cryst. Solids 55, 413 (1983).

${ }^{37}$ P. Jund and R. Jullien, J. Chem. Phys. 113, 2768 (2000).

${ }^{38}$ O. Pilla, L. Angelani, A. Fontana, J. R. Gonçalves, and G. Ruocco, J. Phys.: Condens. Matter 15, S995 (2003).

${ }^{39}$ R. J. Hemley, C. Meade, and H. K. Mao, Phys. Rev. Lett. 79, 1420 (1997).

${ }^{40} \mathrm{C}$. Alba-Simionesco and B. Frick (private communication).

${ }^{41}$ U. Buchenau, M. Prager, N. Nücker, A. J. Dianoux, N. Ahmad, and W. A. Phillips, Phys. Rev. B 34, 5665 (1986).

${ }^{42}$ V. Hizhnyakov, A. Laisaar, J. Kikas, A. Kuznetsov, V. Palm, and A. Suisalu, Phys. Rev. B 62, 11296 (2000). 